\title{
Case - Uretero-internal iliac artery fistula presenting with multiple negative angiographic studies
}

Gautamn Sarwal, $\mathrm{MD}^{1}$; Samir Bidnur, MD $^{2}$; Edmund C.P. Chedgy, MBBS MSc FRCS (Urol); Alex Kavanagh, MD MPH FRCPC ${ }^{3}$

${ }^{1}$ PGY-2 Vascular \& Endovascular Surgery, University of British Columbia; ${ }^{2}$ PGY-4 Urological Surgery, University of British Columbia; ${ }^{3}$ Department of Urological Sciences, Vancouver General Hospital; Vancouver, BC, Canada

Cite as: Can Urol Assoc J 2018 Feb. 6; Epub ahead of print. http://dx.doi.org/10.5489/cuaj.4758

Published online February 6, 2018

$* * *$

\section{Introduction}

We report a rare case of a patient presenting with visible, unexplained hematuria and share the diagnostic challenges faced in the setting of multiple angiographic studies that failed to demonstrate an uretero-internal iliac artery fistula.

Uretero-arterial fistulas (UAF) are rare, but well-recognized, with increasingly common risk factors (Table 1$).{ }^{1}$ Due to its rarity, delay to diagnosis is common, when investigating recurrent hematuria with multiple negative investigations. ${ }^{1,2}$

\section{Case presentation}

A 66-year-old female with chronic ureteric stenting, presented with a complex urologic history including previous chemotherapy and radiation therapy for cervical cancer. As a result of radiotherapy, she had a persistent left ureteric stricture requiring stenting. She was transferred from a community hospital with intermittent, visible hematuria, urosepsis and a chronic perinephric abscess. Based on chronic stenting, a UAF was suspected, but arterial extravasation was not identified on multiple computed tomography arteriograms (CTA), renal or pelvic arteriograms. To manage this unexplained hematuria, renal embolization and a nephrectomy, were performed.

The operation was technically difficult due to the perinephric abscess and frozen abdomen, pursuant to her previous oncological treatment. Once the kidney had been mobilized, the ureter was divided and stent removed. At this point, bright red, pulsatile blood was noted from the distal ureteric stump. This was initially controlled with suture ligation of the ureteric stump, however, the patient became hemodynamically unstable. Intraoperative cystoscopy revealed arterial bleeding from the left ureteral orifice. The patient was subsequently transferred to the radiology suite for pelvic arteriography where, without a tamponading stent, fistulation between the left IIA and ureter was identified. 
The left IIA was selected and the fistula embolized both proximally and distally using $4 \mathrm{~mm} \times 14 \mathrm{~cm}$ Cook MicroNester coils. To completely exclude the fistula, the left IIA orifice was covered with a $8 \mathrm{~mm} \times 50 \mathrm{~mm}$ Gore Viabahn ${ }^{\mathrm{TM}}$ self-expanding covered endovascular stent. With complete exclusion of the fistula and IIA, we noted immediate cessation of hematuria.

\section{Discussion}

Uretero-arterial fistulas (UAF) are well-recognized but uncommon with literature limited to case reports and single centre retrospective reviews. A recent systematic review identified 139 cases of UAF, with a fistula between the IIA and ureter being vanishingly rare. ${ }^{1}$ Fistulas are more common at the pelvic brim, where the ureter crosses the common iliac artery. ${ }^{1} \mathrm{UAF}$ should be included in the differential diagnosis of patients with unexplained visible hematuria presenting with a history of chronic ureteric stenting, pelvic radiotherapy, genitourinary or vascular surgery, all of which have been demonstrated as risk factors (Table 1) predisposing to UAF formation., ${ }^{1,2}$

In some cases of UAF, the initial bleed has been during stent change. ${ }^{1}$ In most cases, the only symptom is painless hematuria. ${ }^{1}$ Massive hematuria has been reported in upto $71 \%$ of patients. ${ }^{1}$ Mortality rates can be as high as $58 \%$ in patients with a missed diagnosis. ${ }^{1}$ The diagnosis is often delayed due to its intermittent nature and subsequent difficulty in demonstrating a fistulous connection. ${ }^{1,2}$ Cystoscopy and retrograde pyelography were historically believed to have the greatest sensitivity, between $45 \%$ and $60 \%$, however with significant bleeding, these may not be possible and therefore sensitivity is believed to be decreased. ${ }^{2,3}$ Ureteroscopy is relatively contraindicated due to the risk of disrupting an obstructing clot or tearing the fistula, resulting in significant hemorrhage. ${ }^{2,3}$

CTAs have a low sensitivity given a fibrotic and scarred pelvis whereas renal arteriography has a sensitivity of upto $41 \%$ for detecting UAFs but as low as $23 \%$ with an insitu ureteric stent. ${ }^{1,3}$ Angiographic findings can be subtle or blatantly obvious at demonstrating a connection between the ureter and artery. ${ }^{1}$ The most obvious finding includes an arterial pseudoaneurysm or gross extravasation of contrast into the ureter. ${ }^{1}$ Subtle findings include irregularities of the vessel especially where the ureter crosses over the vessel. ${ }^{1}$ In some cases, the artery may appear normal. This is likely true when a tamponading ureteric stent remains insitu as the stent may obstruct the fistula to the artery. ${ }^{1}$

Provocative angiography has a demonstrated a sensitivity between $63 \%$ and $100 \% .{ }^{3}$ This technique involves manipulation of the stent under visualization to assess for contrast extravasation. However, we recommend this be performed in a hybrid operating suite because of the risk of hemorrhage requiring emergent surgery or endovascular rescue. A negative test however does not exclude the presence of an UAF either. It therefore requires a high index of suspicion and should be suspected in patients with persistent hematuria and risk factors for $\mathrm{UAF}^{3}$ When unrecognized, attempts at treatment have been directed at a presumed renal source resulting in unnecessary renal embolization and nephrectomy, such as in our case. ${ }^{1}$ Endovascular management of UAF remains favorable given hostile pelvic anatomy and provides immediate 
hemorrhage control. ${ }^{3,4}$ Direct surgical repair also carries a high risk of causing enterotomies and further enteral fistulae. ${ }^{3,4}$

Our patient presented with severe hematuria secondary to a ureteric-internal iliac artery fistula. Her risk factors included previous pelvic radiation and surgery and chronic ureteric stenting. She had multiple negative angiographic investigations, and as such, was believed to have a renal source for her persistent hematuria. Consequently, this lead to renal embolization and nephrectomy. In retrospect, given her risk factors and our suspicion for an UAF, provocative angiography could have been performed in a hybrid operating suite to make the diagnosis. This may have prevented unnecessary renal embolization and nephrectomy.

Once again, this case highlights the need for further awareness of this pathology given high morbidity and mortality and its inherent diagnostic and treatment challenges.

\section{Learning points}

- UAF should be considered in the differential for unexplained hematuria especially in patients with risk factors including chronic ureteric stenting, atherosclerosis, pelvic radiotherapy or genitourinary surgery;

- Negative cross-sectional and angiographic imaging does not necessarily exclude this diagnosis;

- Where a diagnosis of UAF is suspected but remains elusive, provocative angiography without a tamponading stent, may confirm the diagnosis but should be performed in a hybrid operating suite with immediate surgical support. 


\section{References}

1. Van der Bergh RCN, Moll FL, de Vries JP, Lock TMTW. Arterioureteral fistulas: unusual suspects - systematic review of 139 cases. J Urol 2009; 74:251-255.

2. Aslam MZ, Kheradmund F, Patel N, Cranston D. Uretero-iliac artery fistula: a diagnostic and therapeutic challenge. Adv Urol 2010.

3. Krambeck AE, DiMarco DS, Gettman MT, Segura JW. Ureteroiliac artery fistula: diagnosis and treatment algorithm. Urology 2005; 66(5):990-994.

4. Hirsch LM, Amirian MJ, Hubosky SG et al. Urologic and endovascular repair of a uretero-iliac artery fistula. Cdn J Urol 2015; 22(1):7661-5. 


\section{Figures and Tables}

Fig. 1. (A) Left renal arteriogram performed to rule out renal origin of persistent hematuria. Findings: No arterial contrast extravasation. (B) Digital substraction angiogram demonstrating successful embolization of the left main renal artery using 300-500 um embospheres for presumed renal based hematuria.
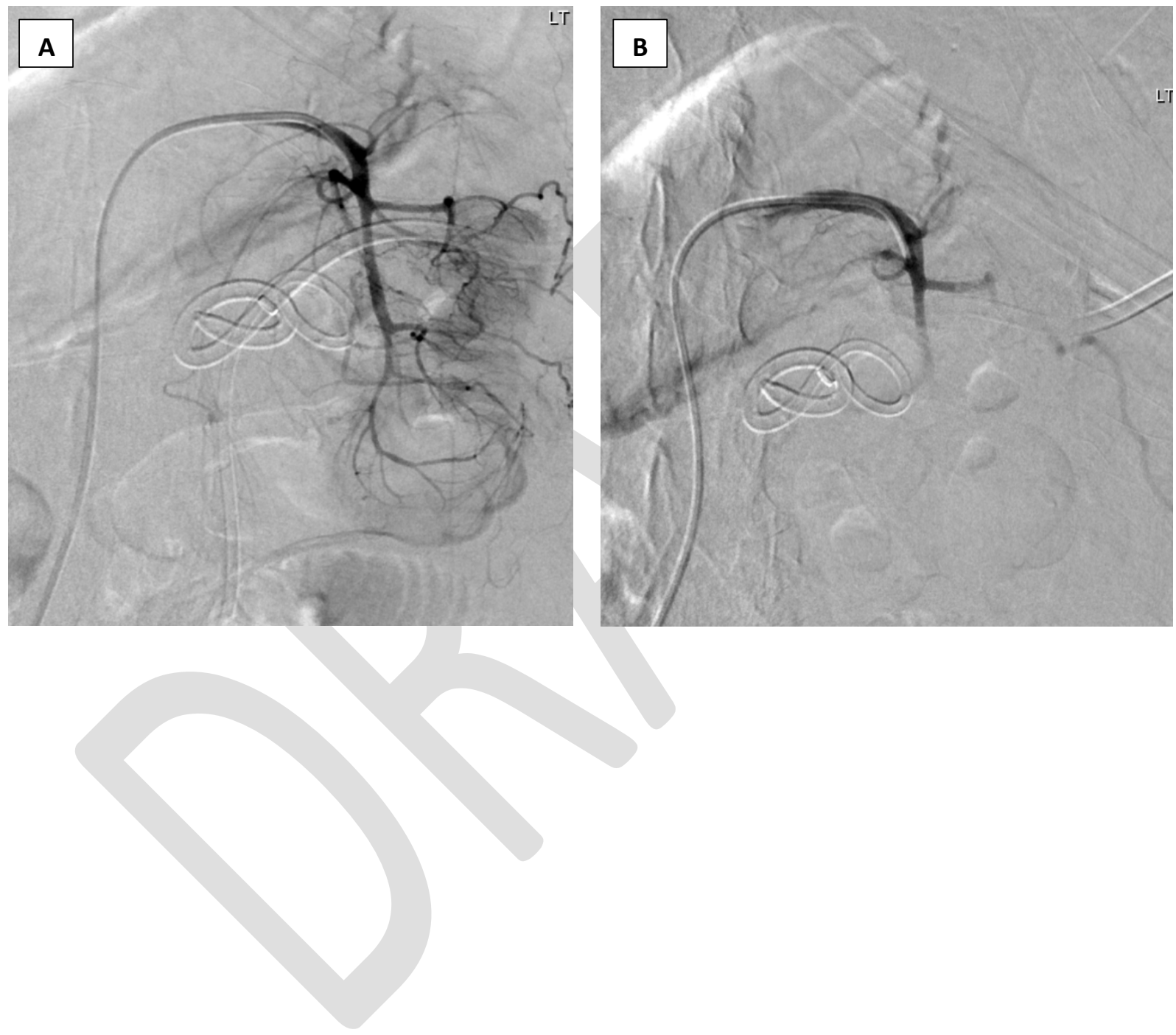
Fig. 2. Left renal arteriogram performed post ureteric stent removal during nephrectomy. (A) Findings: Active contrast extravasation (arrow) from left IIA demonstrating an uretero-internal iliac artery fistula. (B) Trap based microcoil embolization (arrows) to exclude the UAF. (C) Fluoroscopic spot image demonstrating successful deployment of a 8x50mm Gore Viabahn selfexpanding covered stent (arrow) across the left IIA orifice to further exclude the fistula and achieve hemorrhage control. (D) Digital subtraction angiography of the iliac arteries demonstrating complete exclusion of the UAF with both coil embolization and covered stenting.

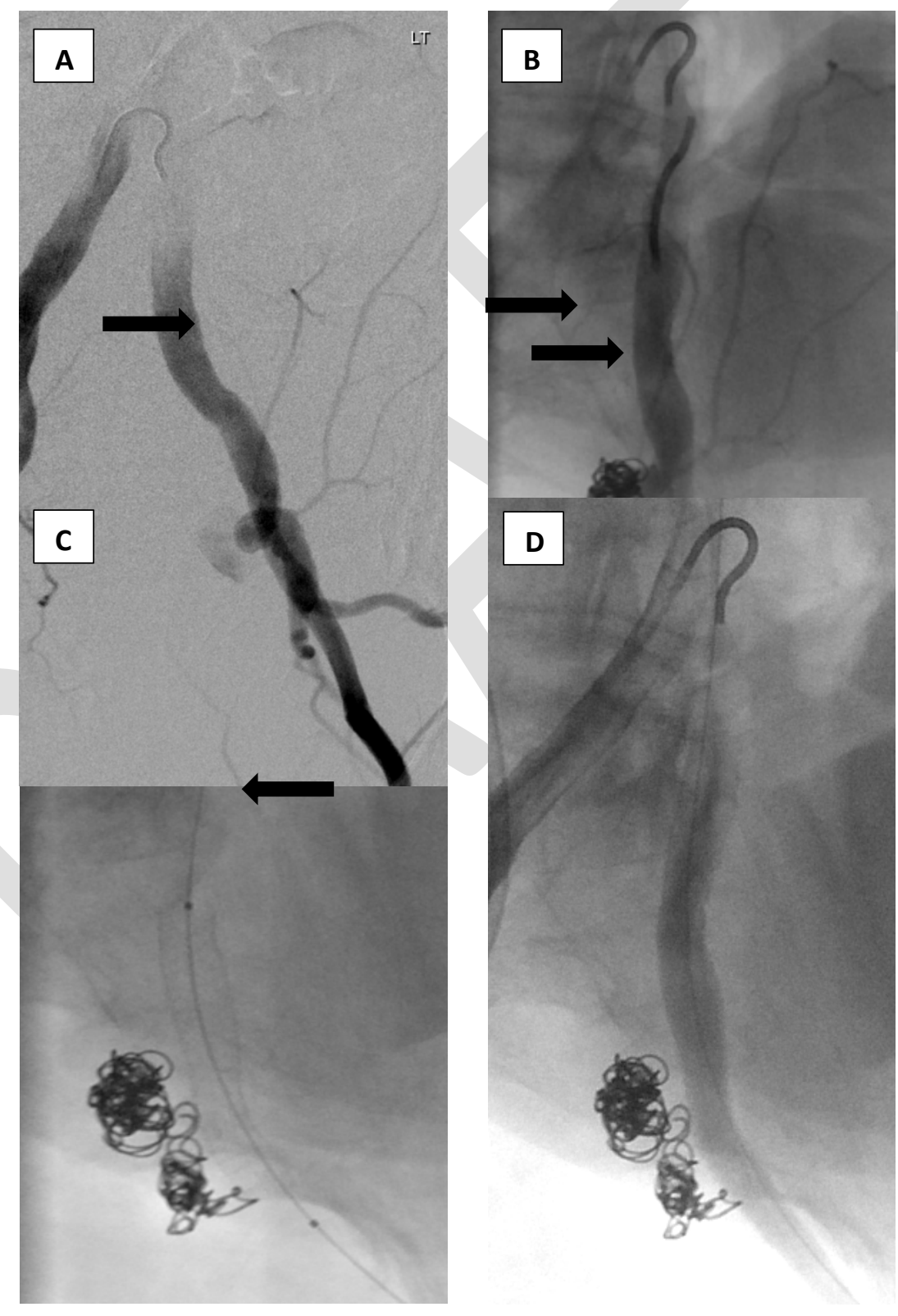


Table 1. Risk factors commonly associated with formation of a uretero-arterial fistula (UAF)

Chronic ureteric stenting

Pelvic radiotherapy

Genitourinary or pelvic surgery

Pelvic arterial reconstructive surgery

Peripheral arterial disease

Pregnancy

Aberrant vascular pathology 